\title{
Evaluación física del huevo comercial de gallinas criollas (Gallus gallus domesticus) en el cantón La Troncal - Ecuador
}

\author{
Physical evaluation of commercial eggs from Creole hens \\ (Gallus gallus domesticus) in La Troncal canton - Ecuador
}

\author{
José Humberto Vera Rodríguez ${ }^{1}$, Rafael Seleyman Lazo Sulca ${ }^{2}$ \\ Gustavo Adolfo Hidalgo Bravo ${ }^{3}$, Cristhian Paul Mendía Mendía ${ }^{4}$ \\ Rosa Hermelinda Naranjo Godoy ${ }^{5}$, Rinna Paola Ortiz Duchi ${ }^{6}$ \\ Stefanie Mercedes Rivera Vásquez ${ }^{7}$, Karen Jordania Moncayo Caravallo ${ }^{8}$ \\ Iralda Narcisa Bravo Gavilanez ${ }^{9}$
}

\section{Resumen}

Se llevó a cabo una investigación descriptiva con un enfoque mixto, con el objetivo de evaluar la calidad del huevo comercial de gallinas criollas provenientes del cantón La Troncal - Ecuador, provincia de Cañar, Ecuador por medio de un estudio físico interno y externo a 1200 huevos (parroquia La Troncal, 400 huevos; parroquia Manuel de J. Calle, 400 huevos y parroquia Pancho Negro, 400 huevos). Cada parroquia fue considerada como un tratamiento, los promedios de las variables del estudio físico externo e interno fueron comparados usando la prueba de Tukey $(p \leq 0.05)$. El color más frecuente fue el azul, con 484 huevos. Resultando más usual la categoría medianos en base al peso de los huevos. Las gallinas de la parroquia La Troncal originaron huevos con mayor porcentaje de cáscara, $\mathrm{pH}$ en la clara, altura de clara y altura de la yema; la parroquia Manuel J. Calle produjo huevos con mayor peso, grosor de la cáscara en el polo angosto, peso de la yema y diámetro de la yema; mientras que la parroquia Pancho Negro produjo huevos con mayor intensidad de la yema, $\mathrm{pH}$ de la yema e índice de la clara. En conclusión, el cantón La Troncal comercializa huevos criollos

1 Magíster en Zootecnia. Docente Investigador Carrera Biotecnología. Universidad Estatal de Milagro. Milagro - Ecuador. Correo: humbertorichi@hotmail.com, ORCID: https://orcid.org/o0oo-0003-3027-059X

2 Magíster en Gerencia en Tecnologías de la Información. Docente Carrera Biotecnología. Universidad Estatal de Milagro. Milagro - Ecuador. Correo: rlazos@unemi.edu.ec, ORCID: https://orcid.org/oooo-0001-5570-0870

3 Magíster en Zootecnia. Docente. Instituto Superior Tecnológico Enrique Noboa Arízaga. La Troncal - Ecuador. Correo: turc01987@ hotmail.com, ORCID: https://orcid.org/o0oo-0001-7855-6402

4 Tecnólogo Superior en Producción Agropecuario. Investigador. Instituto Superior Tecnológico Enrique Noboa Arízaga, La Troncal - Ecuador. Correo: mendiapaul30@yahoo.com, ORCID: https://orcid.org/o0oo-0003-0962-5069

5 Tecnólogo Superior en Producción Agropecuario. Investigador. Instituto Superior Tecnológico Enrique Noboa Arízaga, La Troncal -Ecuador. Correo: rosanaranjogodoy@gmail.com, ORCID: https://orcid.org/o00o-0002-1637-4264

6 Tecnólogo Superior en Producción Agropecuario. Investigador. Instituto Superior Tecnológico Enrique Noboa Arízaga. La Troncal -Ecuador. Correo: luriet.klever@gmail.com, ORCID: https://orcid.org/oooo-0002-2069-5992

7 Tecnólogo Superior en Producción Agropecuario. Investigador. Instituto Superior Tecnológico Enrique Noboa Arízaga. La Troncal - Ecuador. Correo: arctica@outlook.es, ORCID: https://orcid.org/oooo-ooo1-5678-1852

8 Tecnólogo Superior en Producción Agropecuario. Investigador. Instituto Superior Tecnológico Enrique Noboa Arízaga. La Troncal - Ecuador. Correo: jordaniamoncayo1@gamail.com, ORCID: https://orcid.org/o0oo-0001-8802-6085

9 Tecnólogo Superior en Producción Agropecuario. Investigador. Instituto Superior Tecnológico Enrique Noboa Arízaga. La Troncal - Ecuador. Correo: narcisa.1966.b@gmail.com, ORCID: https://orcid.org/0000-0002-6506-5732

Recibido: 13/o9/21 - Aprobado: 22/11/21 
medianos, con variado color, mayormente azules, con aceptable diámetro y altura de la clara, buena pigmentación de la yema, produciendo huevos de muy buena calidad.

Palabras clave: calidad, gallina criolla, huevo.

\section{Abstract}

A descriptive research with a mixed approach was carried out with the objective of evaluating the quality of the commercial egg of Creole hens from La Troncal canton - Ecuador, province of Cañar, Ecuador by means of an internal and external physical study of 1200 eggs (La Troncal parish, 400 eggs; Manuel de J. Calle parish, 400 eggs and Pancho Negro parish, 400 eggs). Each parish was considered as a treatment, the averages of the external and internal physical study variables were compared using Tukey's test $(p \leq 0.05)$. The most frequent color was blue with 484 eggs. The medium category was the most common based on egg weight. Hens from La Troncal parish produced eggs with higher shell percentage, egg white $\mathrm{pH}$, egg white height and yolk height; Manuel J. Calle parish produced eggs with higher egg weight, shell thickness at the narrow pole, yolk weight and yolk diameter; while Pancho Negro parish produced eggs with higher yolk intensity, yolk $\mathrm{pH}$ and egg white index. In conclusion, La Troncal canton commercializes medium-sized Creole eggs, with varied color, mostly blue, acceptable yolk diameter and yolk height, good yolk pigmentation, producing eggs of very good quality.

Keywords: quality, creole hen, egg.

\section{Introducción}

Ecuador se caracteriza por poseer gallinas criollas de traspatio, las cuales ponen huevos muy atractivos por sus variados colores que bajo un sistema de crianza natural poseerían atributos, dentro de sus características y composición, que ameritan ser analizados para garantizar al consumidor un producto de calidad, con grandes beneficios nutricionales que hoy en día están siendo amenazados por la invasión de líneas genéticas comerciales dentro de la producción avícola de postura. Respecto a este contexto, esta investigación evalúa la calidad del huevo comercial de gallinas criollas, provenientes del cantón La Troncal - Ecuador.

Estas gallinas permiten al productor ahorrar recursos, ya que no necesitan una gran inversión para infraestructura, su alimentación está dada mayoritariamente por gusanos, insectos, pasto, frutas, desechos orgánicos de los hogares, granos; siendo así, la principal fuente de proteína animal para personas que viven en zonas rurales y zonas urbano-marginales. 
En Ecuador, el 12 \% de la producción de aves de traspatio, en especial de huevos criollos, es destinada para venta, mientras que el $88 \%$ para autoconsumo, debido al bajo incentivo hacia el productor para producir razas de aves autóctonas, que son material genético del cual se puede obtener ciertas características como la rusticidad.

El huevo de gallina criolla se caracteriza por poseer un mayor contenido de vitamina A, D y E, omega 3 y de proteína, a diferencia con huevos de ponedoras comerciales. Se destaca por su particular coloración de la yema, un color más intenso debido a los pigmentos tomados de su alimentación natural, lo que asegura un mejor sabor.

La calidad del huevo es un indicativo para el consumidor a la hora de comprar, él mismo podría identificar la buena o mala calidad y determinar su adquisición o no. En la actualidad existen normas de calidad para los huevos, tanto internas como externas, sin embargo, los productores y comerciantes hacen caso omiso a las mismas, poniendo en riesgo su producto y la salud de los consumidores.

\section{Revisión de literatura}

La gallina criolla (Gallus gallus domesticus) es una especie de ave distribuida mundialmente y que, desde las evidencias arqueológicas encontradas en Pakistán ${ }^{10}$, Asia, se dice que inició su domesticación a partir del gallo rojo selvático, con algo de hibridación del gallo salvaje gris, su domesticación fue aproximadamente hace siete mil años A.C., con origen múltiple a partir de varias subespecies de Gallus gallus, posteriormente llega a América, con Cristóbal Colón, quien introdujo gallinas provenientes de Asia (Montes et al., 2019).

En Ecuador existen varios tipos de avicultura ${ }^{11}$, pero dentro de ella se caracteriza la de producción de gallinas criollas o de traspatio, con gran importancia zootécnica, ya que son la principal fuente de alimentos para las familias campesinas, generándoles un sustento económico con su excedente de producción, a pesar de tener niveles bajos de producción anuales (Enrique et al., 2016).

El impacto ambiental de la producción de huevos agroecológicos es menor que la producción de huevos convencionales, debido al tipo de dieta basada en alimentos orgánicos producidos en el lugar de crianza, donde se aprovechan los desechos y residuos generados por otro tipo de producciones con fuentes nutricionales alternativas (González et al., 2020).

\footnotetext{
10 Ubicado al sur del continente asiático y colinda al suroeste con Irán, al noroeste con Afganistán, al noreste con China, al este con la India, y al sur con el océano Índico.

11 Gallinocultura, meleagricultura, anacultura,coturnicultura, numidicultura, colombicultura, colombofilia, estrutiocultura, canaricultura.
} 
El huevo es un alimento que genera ingresos económicos a las familias que se dedican a la producción de esta actividad (Gutiérrez et al., 2007), además, aporta proteína de alta calidad y numerosos nutrientes con potenciales beneficios para la salud (Dussaillant et al., 2017). Teniendo en cuenta la alta calidad nutritiva del huevo, junto con su contribución de numerosos componentes bioactivos benéficos para el bienestar humano, este alimento puede ser considerado un constituyente importante dentro de la dieta de personas en todas las etapas del ciclo vital; pero, podría jugar un rol aún más significativo en etapas de mayor demanda nutricional como la infancia o el embarazo, o en aquellos más vulnerables como los adultos mayores (Porbén 2008; Quitral et al., 2009).

La inocuidad alimentaria es un tema que preocupa a las poblaciones en el ámbito mundial, existiendo varias investigaciones que evidencian alarmantes resultados en el ámbito de control de calidad de los alimentos, generando problemas de salud pública. Por lo tanto, se deben establecer medios seguros para poder diagnosticar y evaluar la inocuidad de los alimentos (Ulloa et al., 2020).

La vida útil del huevo dependerá de un sinnúmero de factores, entre ellos, el tiempo de almacenamiento y temperatura ambiente que afecta las medidas de calidad, hay que tener presente que desde el momento en que la gallina pone el huevo hasta que este llegue al consumidor final se pueden presentar varios cambios fisicoquímicos que alteran sus características organolépticas (Ángel et al., 2021).

Las aves ocupan un lugar primordial en la alimentación humana (Lozano et al., 2018). Es preciso conocer bien el sistema productivo, tanto en la crianza y comercialización, para poder ofrecer huevos con una calidad adecuada. La genética, edad, muda, nutrición y el estado sanitario del animal desempeñan un papel vital en la calidad del huevo. Además, el período post-puesta, junto con el tiempo y las condiciones de almacenamiento, podrían determinar su calidad (Ortiz y Mallo, 2013).

Respecto a la calidad, existe tecnología que permite identificar estos factores que afectan a los huevos, siendo así las fisuras en la cáscara, problemas asociados a sus condiciones de sostenimiento, alimentación, sistemas de recolección, entre otros (Sánchez y Alfonso, 2019). Además, existen normativas estatales como la Norma Técnica Ecuatoriana (NTE INEN 1973:2013), que establece los requisitos que deben cumplir los huevos comerciales para consumo humano y que en muchos de los casos no se da cumplimiento (Vera et al., 2020). 


\section{Materiales y métodos}

\section{Localización}

El ensayo de la calidad del huevo comercial de gallinas criollas (Gallus gallus domesticus) se realizó en las tres parroquias del cantón La Troncal, de la Provincia de Cañar, Ecuador, geográficamente ubicada en las coordenadas: latitud sur $2^{\circ} 28^{\prime} 22^{\prime \prime}$ y $2^{\circ} 30^{\prime} 05^{\prime \prime}$; longitud oeste $79^{\circ} 14^{\prime} 14^{\prime \prime}$ y $79^{\circ} 31^{\prime} 45^{\prime \prime}$.

Su extensión comprende 32.780 hectáreas, fraccionada con las parroquias: La Troncal 12.483,4 ha, Manuel de J. Calle 3.746,8 ha y Pancho Negro 16.549,8 ha. Posee una altitud entre los 24 y los 200 m.s.n.m., la temperatura media es de $24.6^{\circ} \mathrm{C}$., mínima de $20.9^{\circ} \mathrm{C}$ y máxima de $29.2^{\circ} \mathrm{C}$. (GAD Municipal La Troncal, 2021)

\section{Métodos a seguir}

Se recolectaron 1200 huevos comerciales de gallinas criollas procedentes de las parroquias del cantón La Troncal: La Troncal 400 huevos, Manuel de J. Calle 400 huevos y Pancho Negro 400 huevos.

Para este estudio se determinaron las siguientes variables:

Análisis físico externo: Color del cascarón, peso del huevo (g), clasificación por su peso (Norma Técnica Ecuatoriana NTE INEN 1973:2013), diámetro longitudinal (cm), diámetro transversal (cm), índice morfológico (\%), largo perimetral (cm), ancho perimetral $(\mathrm{cm})$, peso del cascarón $(\mathrm{g})$, porcentaje de cáscara (\%), grosor de la cáscara en el polo ancho $(\mathrm{mm})$, grosor de la cáscara en el polo angosto $(\mathrm{mm})$, grosor de la cáscara en la zona ecuatorial (mm), espesor del cascarón ( $\mathrm{mm}$ ).

Análisis físico interno: Color de la yema, peso de la yema (g), peso de la clara (g), porcentaje de la yema (\%), porcentaje de la clara (\%), $\mathrm{pH}$ de la clara, $\mathrm{pH}$ de la yema, diámetro de la clara $(\mathrm{cm})$, diámetro de la yema $(\mathrm{cm})$, altura de la clara $(\mathrm{cm})$; altura de la yema (cm), índice de la yema (\%), índice de la clara (\%).

Los datos de pesos ( $\mathrm{g}$ ) fueron tomados con una balanza digital marca CAMRY ${ }^{\circledR}$ EK5055, las medidas de longitud ( $\mathrm{mm}$ ) se consiguieron con un micrómetro digital marca FOWLER ${ }^{\circledR} \mathrm{N}^{\circ} 54-815$, calibrado a un rango de hasta $2.5 \mathrm{~cm}$. Las medidas de longitud $(\mathrm{cm})$ se obtuvieron con una cinta métrica flexible marca TAYLOR ${ }^{\circledR}$ y un calibrador análogo marca SURTEK ${ }^{\circledR}$, con una precisión de +-03 mm.

Conseguidos el diámetro longitudinal y el diámetro transversal del huevo, el índice morfológico se logró con la fórmula diámetro transversal×100/diámetro longitudinal. El porcentaje de cáscara se obtuvo con la fórmula peso de cáscara×100/peso de huevo. 
Para determinar la intensidad del color de la yema se requirió del abanico colorimétrico $\left(\mathrm{DSM}^{\circledR}\right)$. El porcentaje de yema fue estimado con la fórmula peso de yema×100/ peso de huevo, el porcentaje de clara se obtuvo con la fórmula peso de clara×100/peso de huevo, para medir el $\mathrm{pH}$ de yema y de clara se emplearon tiras reactivas de $\mathrm{pH}$, el índice de yema se obtuvo con la fórmula altura de yema×100/diámetro de yema y el índice de clara con la fórmula altura de clara×100/diámetro de clara.

Los datos fueron manejados bajo un diseño completamente aleatorizado (DCA), siendo cada parroquia un tratamiento y 400 huevos sus repeticiones, señalando que, cada huevo fue calificado como una unidad experimental. Las medias de las variables del análisis físico externo y del interno, fueron separadas recurriendo a la prueba de Tukey $(p \leq 0.05)$. El paquete estadístico empleado fue InfoStaf versión 2019.

\section{Resultados y discusión}

La tabla 1, muestra los resultados obtenidos del análisis físico interno y externo de los huevos comerciales de gallinas criollas (Gallus gallus domesticus) del cantón La Troncal, Ecuador.

Tabla 1: Comparación de medias de variables en estudio

\begin{tabular}{|c|c|c|c|c|}
\hline \multirow{2}{*}{ VARIABLES } & \multicolumn{3}{|c|}{ PARROQUIAS CANTÓN LA TRONCAL } & \multirow{2}{*}{ Significancia } \\
\hline & La Troncal & Manuel J. Calle & Pancho Negro & \\
\hline \multicolumn{5}{|l|}{ ANÁLISIS FÍSICO EXTERNO } \\
\hline Peso del huevo (g) & $56,86 a$ & $57,94 b$ & $56,89 a$ & * \\
\hline Diámetro longitudinal (cm) & $5,68 b$ & $4,63 a$ & $5,62 b$ & * \\
\hline Diámetro transversal (cm) & $4,26 b$ & $3,18 \mathrm{a}$ & $4,22 b$ & * \\
\hline Índice morfológico (\%) & $75,15 b$ & $69,14 a$ & $75,19 b$ & * \\
\hline Largo perimetral (cm) & $15,74 a$ & $15,89 \mathrm{~b}$ & $15,73 a$ & * \\
\hline Ancho perimetral (cm) & $13,54 a$ & $13,55 a$ & $13,59 a$ & ns \\
\hline Peso del cascarón (g) & $6,54 b$ & $6,45 b$ & $6,11 a$ & * \\
\hline Porcentaje de cáscara (\%) & $11,56 \mathrm{C}$ & $11,22 b$ & $10,81 a$ & ** \\
\hline Grosor cáscara polo ancho (mm) & $0,29 a$ & $0,29 b$ & $0,29 a$ & * \\
\hline Grosor cáscara polo angosto (mm) & $0,28 b$ & $0,29 C$ & $0,27 a$ & ** \\
\hline Grosor cáscara zona ecuatorial (mm) & $0,27 b$ & $0,26 a$ & $0,28 \mathrm{C}$ & $* *$ \\
\hline Espesor del cascarón (mm) & $0,28 \mathrm{a}$ & $0,28 b$ & $0,28 \mathrm{a}$ & * \\
\hline \multicolumn{5}{|l|}{ ANÁLISIS FÍSICO INTERNO } \\
\hline Color de la yema & $11,96 b$ & $9,87 a$ & $13,02 \mathrm{C}$ & $* *$ \\
\hline Peso de la clara (g) & $32,45 b$ & $31,69 a$ & $31,43 a$ & * \\
\hline Peso de la yema (g) & $17,87 a$ & $19,80 \mathrm{C}$ & $19,35 b$ & $* *$ \\
\hline
\end{tabular}




\begin{tabular}{|l|l|l|l|l|}
\hline \multirow{2}{*}{ VARIABLES } & \multicolumn{3}{c|}{ PARROQUIAS CANTÓN LA TRONCAL } & \multirow{2}{*}{ Significancia } \\
\cline { 2 - 4 } & La Troncal & Manuel J. Calle & Pancho Negro & \\
\hline Porcentaje de la clara (\%) & $57,10 \mathrm{~b}$ & $54,58 \mathrm{a}$ & $55,15 \mathrm{a}$ & $*$ \\
\hline Porcentaje de la yema (\%) & $31,32 \mathrm{a}$ & $34,27 \mathrm{~b}$ & $34,07 \mathrm{~b}$ & $*$ \\
\hline pH de la clara & $9,67 \mathrm{c}$ & $8,71 \mathrm{a}$ & $9,20 \mathrm{~b}$ & $* *$ \\
\hline pH de la yema & $8,78 \mathrm{~b}$ & $8,66 \mathrm{a}$ & $9,96 \mathrm{c}$ & $* *$ \\
\hline Diámetro de la clara $(\mathrm{cm})$ & $9,35 \mathrm{~b}$ & $9,92 \mathrm{C}$ & $7,66 \mathrm{a}$ & $* *$ \\
\hline Diámetro de la yema (cm) & $4,33 \mathrm{~b}$ & $4,06 \mathrm{a}$ & $4,29 \mathrm{~b}$ & $*$ \\
\hline Altura de la clara $(\mathrm{cm})$ & $0,66 \mathrm{c}$ & $0,53 \mathrm{a}$ & $0,60 \mathrm{~b}$ & $* *$ \\
\hline Altura de la yema $(\mathrm{cm})$ & $1,69 \mathrm{c}$ & $1,54 \mathrm{a}$ & $1,58 \mathrm{~b}$ & $* *$ \\
\hline Índice de la clara $(\%)$ & $7,14 \mathrm{~b}$ & $5,50 \mathrm{a}$ & $7,89 \mathrm{c}$ & $* *$ \\
\hline Índice de la yema $(\%)$ & $39,28 \mathrm{~b}$ & $38,57 \mathrm{~b}$ & $37,15 \mathrm{a}$ & $*$ \\
\hline
\end{tabular}

a, b y c letras distintas en una misma hilera indican diferencias estadísticas según Tukey (0.05); ns no significativo; * significativo; ** altamente significativo. Fuente: elaboración propia.

De forma general, las gallinas criollas de la parroquia La Troncal produjeron valores altos en el porcentaje de cáscara, $\mathrm{pH}$ de la clara, altura de la clara, altura de la yema, mientras que los valores obtenidos con los huevos de las otras parroquias fueron levemente menores. Sin embargo, las gallinas de la parroquia Manuel J. Calle produjeron promedios altos en el peso del huevo, grosor de la cáscara en el polo angosto, peso de la yema y diámetro de la yema, mientras que las otras parroquias presentaron promedios inferiores. Mientras que las gallinas de la parroquia Pancho Negro presentaron promedios altos en el color de la yema, $\mathrm{pH}$ de la yema, índice de la clara, los valores obtenidos con los huevos de las otras dos parroquias fueron menores.

El mayor peso del huevo encontrado en las gallinas en pastoreo está asociado principalmente a un mayor peso de la yema, aspecto importante debido a que en esta se encuentra la mayor cantidad de sólidos, proteínas y ácidos grasos (Jaramillo et al., 2018).

Las gallinas criollas no cubren sus necesidades diarias de calcio en su dieta común, debido a que no son alimentadas con concentrado ya que es insustentable debido al elevado costo del alimento y a la baja productividad de las gallinas criollas (Rodríguez y Valencia, 2018), por tal motivo, para mejorar el grosor de la cáscara del huevo, hay que suministrar en la dieta carbonato de calcio, especialmente adicionarlo en las últimas horas de la tarde, con un nivel de granulometría de 2 a 4 mm (Rodríguez y Bravo, 2019).

El espesor de la cáscara del huevo está relacionado con la dureza de la misma. El peso de la cáscara se puede medir rompiendo el huevo, separando la cáscara, secándola y expresándolo como porcentaje respecto al peso del huevo. La resistencia de la cáscara no solo depende de su peso y espesor, sino también de la calidad de su estructura. Los 
principales factores que afectan la calidad de la cáscara son genéticos, nutricionales, productivos y relacionados con el estrés (Ortiz y Mallo, 2013).

La calidad del huevo, como el color de la yema, está asociada al consumo de forraje y posiblemente a otros factores ambientales (Jaramillo et al., 2018). El color amarillo de la yema de huevo se debe a las xantofilas, un pigmento que no tiene valor nutricional (De las Moras, M. 2008).

Comercialmente, el mejor color de yema se encuentra entre 7 y 12 unidades de color (INEN, 2013). El pH de la clara y de la yema es un indicativo del grado de frescura de los huevos (Carranco et al., 2017).

García et al. (2016) estudió los huevos de gallinas White Leghorn L33 de 39 semanas, encontrando los siguientes resultados: largo del huevo, $5.58 \mathrm{~cm}$; ancho del huevo, $4.26 \mathrm{~cm}$; altura de clara, $0.69 \mathrm{~cm}$; ancho de clara, $7.18 \mathrm{~cm}$; largo de clara, 9.62 cm; altura de yema, $1.69 \mathrm{~cm}$; diámetro de yema, $4.12 \mathrm{~cm}$; grosor de cáscara, $0.41 \mathrm{~mm}$; índice de clara, 5\%; índice de la yema, 4.1\%; índice morfológico, 75.55\%.

En la clasificación de los huevos por su color, se encontraron 4 categorías respecto a la totalidad de huevos colectados en el Cantón La Troncal, los resultados se observan en la Figura 1.

\section{Color de la cáscara de los huevos de gallinas criollas por parroquias del cantón La Troncal}

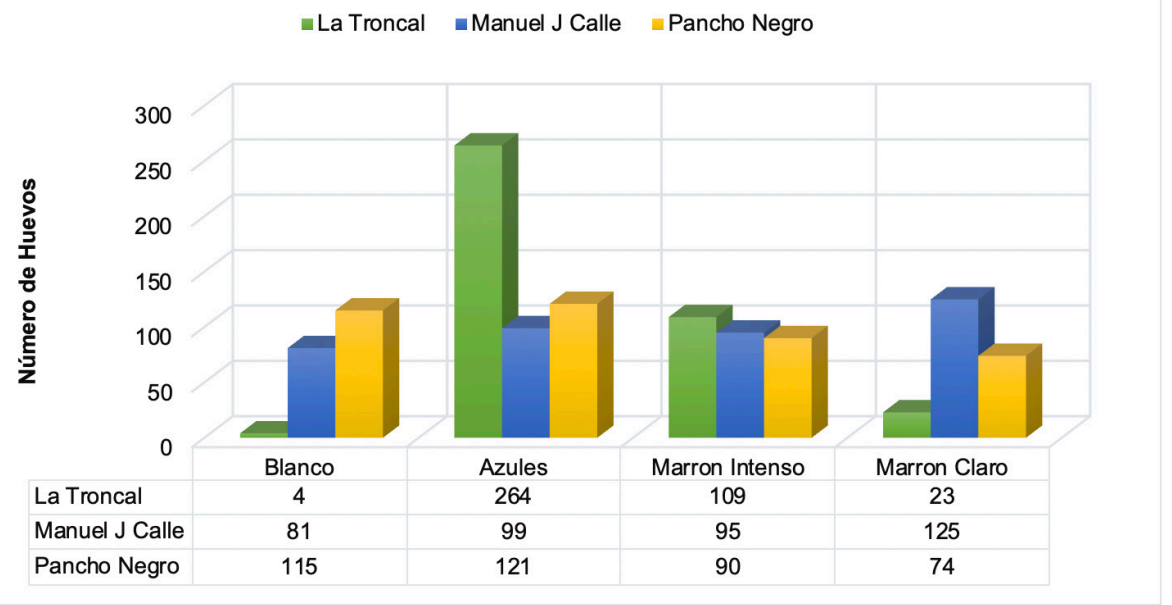

Figura 1: Clasificación de los huevos por el color de la cáscara, por parroquias del cantón La Troncal. Fuente: elaboración propia. 
El análisis muestra que el color más frecuente fue el azul, con 484 huevos; seguido el color marrón intenso, con 294 huevos; el color marrón claro, con 222 huevos y color blanco, con 200 huevos.

Las cáscaras de los huevos de gallina pueden tener distintos colores, aunque los más habituales son los blancos y morenos (pardo claro), el color de la cáscara depende exclusivamente de la especie de la gallina ponedora y no afecta a su calidad, sabor, características al cocinar, valor nutricional o grosor (De las Moras, 2008).

El color de la cáscara de los huevos, se debe al pigmento que predomina sobre la protoporfirina en ellos (Wang et al., 2009). Además, el tiempo de oviposición afecta la intensidad del color de la cáscara, siendo más oscuros los huevos puestos en las primeras horas del día y más claros en horas avanzadas de la mañana, además la intensidad del color disminuye, con la edad de la gallina (Samiullah et al., 2016).

La clasificación de los huevos comerciales de gallinas criollas en el cantón La Troncal, por su peso, según la Norma Técnica Ecuatoriana NTE INEN 1973:2013, se muestra en la Figura 2.

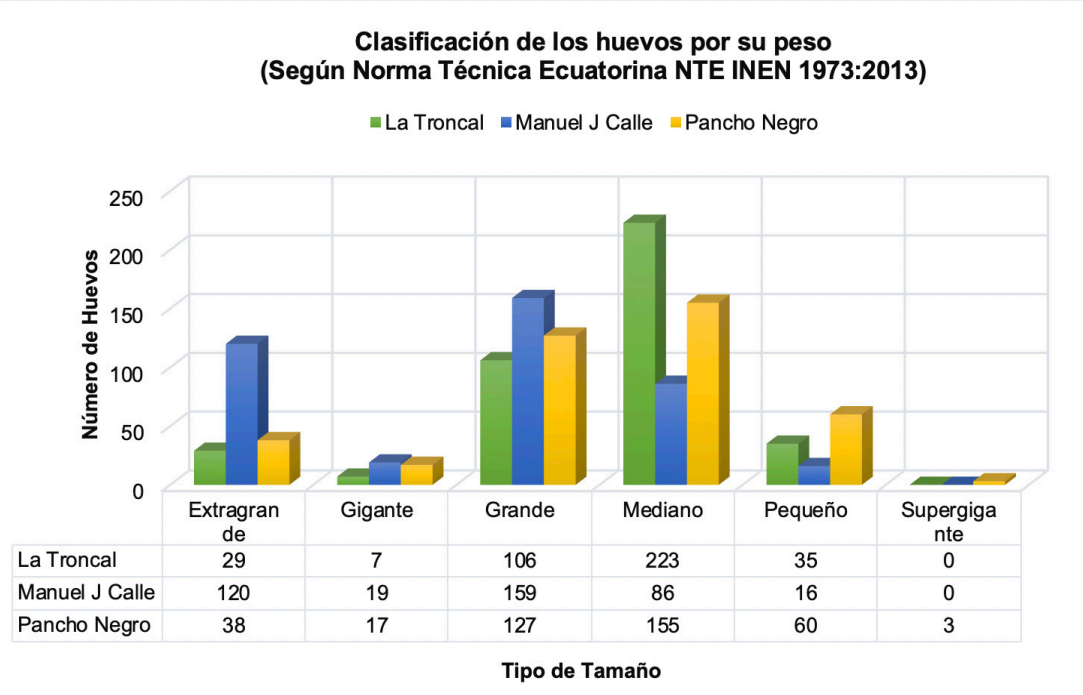

Figura 2: Clasificación de los huevos criollos según Norma Técnica Ecuatoriana NTE INEN 1973:2013, por parroquias del cantón La Troncal. Fuente: elaboración propia.

Se observan, en la Figura 2, seis categorías de peso en las tres parroquias. Respecto a la totalidad de huevos $(1,200)$, la categoría más frecuente fue los medianos $(38,67 \%)$, seguida el grande $(32,67 \%)$, extragrande $(15,58 \%)$, pequeño $(9,25 \%)$, gigante $(3,58 \%)$ y súper gigante $(0,25 \%)$. Es decir, $90,50 \%$ de los huevos fueron de categorías extragrande, 
gigante, grande y mediano. El huevo de traspatio es de tamaño variable, en comparación con el huevo incubable comercial (Escobar et al., 2019).

En las comunidades ecuatorianas se han encontrado hasta 10 biotipos de gallinas criollas (Villacís et al., 2014). Estas presentan diferentes pesos iniciales, aun cuando son de edades similares, lo que ha influenciado en su ganancia de peso y su peso final, dado a diferente medio ambiente donde se desarrollaron, lo que ha conllevado a obtener huevos de diferentes pesos y tamaños (Correa et al., 2007; Toalombo et al., 2019).

El huevo de gallinas criollas forma una parte importante dentro de la nutrición de las familias campesinas (Mayorga et al., 2019; Tovar et al., 2015), presenta dimensiones reducidas, en comparación con el huevo comercial, sin embargo, los indicadores de calidad sugieren que el huevo de gallina de traspatio tiene calidad similar al de gallinas de la estirpe comercial (Juárez et al., 2010; Oñate et al., 2020).

\section{Conclusiones}

El estudio del análisis físico externo y análisis físico interno en las diferentes parroquias estudiadas, produjeron huevos estadísticamente diferentes. Similar trabajo fue realizado por Vera et al. (2020), en gallinas de huevo marrón, encontrando huevos estadísticamente diferentes en las parroquias del cantón La Troncal - Ecuador. Por tanto, los huevos comerciales de gallinas criollas, cumplen con la mayoría de las características reportadas por literatura nacional e internacional, por lo que se pueden considerar como de buena calidad.

\section{Recomendaciones}

Se sugiere clasificar los huevos de gallinas criollas dando cumplimiento a la Norma Técnica Ecuatoriana (NTE INEN 1973:2013), y brindar así un mejor producto al consumidor final, al momento de la compra.

Esta investigación desempeña un papel fundamental en la seguridad alimentaria, con esta base damos a conocer al consumidor final que los huevos que consumen son de buena calidad a pesar que no se da cumplimiento a la Norma Ecuatoriana NTE INEN 1973:2013 para su expendio o comercialización.

\section{Lista de referencias}

Ángel-Isaza, J., Suárez-Orejarena, C., Serrano-Galvis, P., Parra-Mendez, L., CamposParedes, H., y Martínez, B. C. (2021). Evaluación de escala visual como medida de calidad interna y frescura de huevo comercial. Revista MVZ Córdoba, 26(2), 1-7. https://doi.org/10.21897/rmvz.2031 


\section{AGROPECUARIA}

Carranco, J.M.E., Carrillo, D.S., Ávila G.E., y Solano, M.D.L. (2017). Cambios de la fracción hidrosoluble de huevo de gallinas alimentadas con harina de camarón almacenado a diferentes tiempos y temperaturas. Revista Mexicana de Ciencias Pecuarias, 8(4), 365-373. https://doi.org/10.22319/rmcp.v8i4.4183

Correa, J. S., Salas, M. J., Franco, L. S., y Ricalde, R. S. (2007). Indicadores de producción de huevo de gallinas criollas en el trópico de México. Archivos de Zootecnia, 56(215), 309-317. http://www.redalyc.org/articulo.oa?id=49521504

De las Moras, M.C R. (2008). Alteraciones de la cáscara, clara y yema de huevo. Ganadería, 52, 56-57. https://www.mapa.gob.es/ministerio/pags/biblioteca/ revistas/pdf_Ganad/Ganad_2008_52_56_57.pdf

Dussaillant, C., Echeverría, G., Rozowski, J., Velasco, N., Arteaga, A., y Rigotti, A. (2017). Consumo de huevo y enfermedad cardiovascular: una revisión de la literatura científica. Nutrición Hospitalaria, 34(3), 710-718. http://dx.doi. org/10.20960/nh.473

Enrique, V. R. G., Sánchez, G. E., Castillo, F. C., y Agusto, R. L. (2016). Características fenotípicas de las gallinas criollas de comunidades rurales del sur del Ecuador. Centro de biotecnología, 3(1). http://revistas.unl.edu.ec/index.php/biotecnologia/ article/view/30

Escobar, M. A. C., Barradas, A. V., Salas, M. P. J., López, J. C. G., Garrido, S. J. L., Bernal, E. I. S., y Serrano, N. Y. A. (2019). El huevo de traspatio: características físicas y desempeño en pruebas de incubación artificial. Acta Universitaria, 29(1), 1-16. https://doi.org/10.15174/au.2019.2381

GAD Municipal La Troncal. (2021). Datos generales ciudad La Troncal. [En Línea]. http://www.latroncal.gob.ec/WEB17/VARIOS/CIUDAD.PHP (Accedido 12-septiembre-2021).

García, D. M., Colas, M. C., López, W. S., Pérez, E. O., Sánchez, A. P., Lamazares, M. C., y Grandía, R. G. (2016). El peso corporal y su efecto sobre indicadores bioproductivos en gallinas White Leghorn L33. Revista de la Facultad de Medicina Veterinaria y de Zootecnia, 63(3), 188-200. http://dx.doi.org/10.15446/rfmvz. v63n3.62714

González-Velandia, K. D., Landázury-Correa, A., y Chaparro, A. M. (2020). Evaluación de impactos ambientales en la cadena de producción de huevos agroecológicos con un enfoque de ciclo de vida. Revista de Ciencias Ambientales, 54(2), 165-179. http://doi.org/10.15359/rca.54-2.9 
Gutiérrez-Triay, M. A., Segura-Correa, J. C., López-Burgos, L., Santos-Flores, J., Ricalde, R. H. S., Sarmiento-Franco, L., y Molina-Canul, G. (2007). Características de la avicultura de traspatio en el municipio de Tetiz, Yucatán, México. Tropical and subtropical Agroecosystems, 7(3), 217-224. http://www.redalyc.org/articulo. oa?id=93970308

INEN N. (2013). Huevos comerciales y ovoproductos. Requisitos. https://www.normalizacion.gob.ec/buzon/normas/nte_inen_1973-2.pdf (Accedido 12-septiembre-2021).

Jaramillo, Á. H., Mojica, J., Caro, É. A., y Sosa, J. (2018). Evaluación de la calidad del huevo de gallina en dos sistemas de alojamiento-piso convencional con suplementación de sauco (Sambucus nigra) y pastoreo con kikuyo (Pennisetum clandestinum)-en la Sabana de Bogotá. Revista Siembra CBA, 1, 59-77. http:// revistas.sena.edu.co/index.php/Revsiembracba/article/download/1881/1987

Juárez, C.A., Gutiérrez, V.E., Segura, C.J., y Santos R.R. (2010). Calidad del huevo de gallinas criollas criadas en traspatio en Michoacán, México. Tropical and subtropical agroecosystems, 12(1), 109-115. http://www.redalyc.org/articulo. oa?id=93913074011

Lozano, N. A., Asencio, M. G., Marín, R. D., \& Yucailla, V. A. (2018). Producción de pollos criollos con una incubadora artesanal de huevos en la comuna San Vicente cantón Santa Elena. Revista Científica y Tecnológica UPSE, 5(1), 90-95. https://doi.org/10.26423/rctu.v5i1.336

Mayorga, I. C., Bravo, A. Z., Gomez, K. P., Parra, E. P., García, D. C., Lozano, N. V. A., y Verónica, A. Y. (2019). Animales domésticos de traspatio en el bosque deciduo de tierras bajas de la Comuna San Marcos parroquia Colonche. Revista Amazónica Ciencia y Tecnología, 8(2), 202-208. https://dialnet.unirioja.es/servlet/ articulo? codigo $=7153089$

Montes, D., De La Ossa, J., y Hernández, D. (2019). Caracterización morfológica de la gallina criolla de traspatio de la subregión Sabana departamento de Sucre (Colombia). Revista MVZ Córdoba, 24(2), 7218-7224. https://doi.org/10.21897/ rmvz.1646

Oñate, F. J., Gavilanes, A. A. V., \& Calle, O. E. B. (2020). Calidad de huevos de gallinas criollas criadas en traspatio en Macas, Ecuador. Dominio de las Ciencias, 6(3), 662-673. http://dx.doi.org/10.23857/dc.v6i3.1307

Ortiz, Á., \& Mallo, J. J. (2013). Factores que afectan a la calidad externa del huevo. Albéitar, 170, 18-19. https://norel.net/en/system/files/factores_que_afectan_a_ la_calidad_del_huevo.pdf 
Porbén, S. S. (2008). El huevo como aliado de la nutrición y la salud. Revista Cubana de Alimentación y Nutrición, 18(2), 15. http://revalnutricion.sld.cu/index.php/ rcan/article/view/919

Quitral, V., Donoso, M. L., y Acevedo, N. (2009). Comparación físico-química y sensorial de huevos de campo, orgánicos y comerciales. Revista Salud Pública y Nutrición, 10(2). https://www.medigraphic.com/cgi-bin/new/resumen. cgi? IDARTICULO $=22528$

Rodríguez, D., y Valencia, F. (2018). Reducción de los costos alimenticios de gallinas criollas ponedoras con suplementación de larva de mosca (Musca domestica L.) en La Granja Agroecológica El Mirador Guacarí Valle del Cauca. Revista Colombiana de Zootecnia, 4(7), 20-24. http://anzoo.org/publicaciones/index. $\mathrm{php/anzoo/article/view/25}$

Rodríguez, J. H. V., y Bravo, G. A. H. (2019). El Efecto de diferentes niveles de suministro de carbonato de calcio sobre el peso y grosor de la cáscara del huevo. Revista Colombiana de Ciencia Animal-RECIA, 11(2), 719-719. https://doi.org/10.24188/ recia.v11.n2.2019.719

Samiullah, S., Roberts, J., y Chousalkar, K. (2016). Oviposition time, ûock age, and egg position in clutch in relation to brown eggshell color in laying hens. Poultry Sci, 95, 2052-2057. https://doi.org/10.3382/ps/pew197

Sánchez, I. C. N., y Alfonso, J. N. M. (2019). Revisión: estimación de deficiencias en la calidad del huevo. Publicaciones e Investigación, 13(1), 103-110. https://doi. org/10.22490/25394088.2362

Toalombo, P. A., Navas G.F.J., Andrade Y.V.C., Trujillo, J.V., Martinez, J., y Delgado, J. V. (2019). Caracterización productiva y organoléptica de huevos de gallinas de campo de la región sierra del Ecuador. Archivos de zootecnia, 68(263), 412-415. https://www.universidaddecordoba.eu/ucopress/az/index.php/az/article/ view/4201

Tovar-Paredes, J. L., Narváez-Solarte, W., y Agudelo-Giraldo, L. (2015). Tipificación de la gallina criolla en los Agroecosistemas campesinos de producción en la zona de influencia de la selva de Florencia (Caldas). Revista Luna Azul, 41, 5772. http://dx.doi.org/10.17151/luaz.2015.41.4

Ulloa, J. O., Arteaga, E. M. C., Avilés, A. M. O., \& Moscoso, S. P. D. (2020). Revisión sistemática de estudios sobre inocuidad alimentaria en Cuenca, Ecuador, periodo 1981-2017. Segurança Alimentar e Nutricional, 27, 1-12. http://dx.doi. org/10.20396/san.v27io.8654199 
Vera, R.J.H., Cepeda, L.W.E., Torres, A.K.M., Bueno, G.E.K., Mendoza, L.C.A., Merchan P.B. T., y Rivera, R.D.M. (2020). Evaluación de la calidad del huevo marrón comercial del cantón La Troncal, Ecuador. Revista Colombiana de Ciencia AnimalRECIA, 12(2), 771. https://doi.org/10.24188/recia.v12.n2.2020.771

Villacís, G., Escudero, G., Cueva, F., y Luzuriaga, A. (2014). Características morfométricas de las gallinas criollas de comunidades rurales del sur del Ecuador. Rev Inv Vet Perú, 27, 218-224. https://doi.org/10.15381/rivep.v27i2.11639

Wang, X., Zhao, C., Li, J., Xu, G., Lian, L., Wu, C., y Deng, X. (2009). Comparison of the total amount of eggshell pigments in Dongxiang brown-shelled eggs and Dongxiang blue-shelled eggs. Poultry Sci, 88, 1735-1739. https://doi.org/10.7717/ peerj.3706 